\title{
LOS ESPACIOS DE LA LOCURA EN LA TOSCANA DEL SIGLO XVIII: ESTRATEGIAS Y NEGOCIACIONES PARA ENFRENTAR LA ENFERMEDAD MENTAL
}

\author{
Mariana Labarca Pinto \\ Universidad Adolfo Ibáñez \\ marianalabarc@gmail.com \\ ORCID iD: https://orcid.org/0000-0002-5080-3018
}

Recibido: 2 enero 2018; Aprobado 18 octubre 2018.

\begin{abstract}
Cómo citar este artículo/Citation: Labarca Pinto, Mariana (2019), "Los espacios de la locura en la Toscana del siglo XVIII: Estrategias y negociaciones para enfrentar la enfermedad mental”, Asclepio, 71(1): p250. https://doi.org/10.3989/asclepio.2019.02.

RESUMEN: Este trabajo estudia los diferentes espacios en que aparece registrada la locura en el Gran Ducado de Toscana durante el siglo XVIII. A partir de la revisión de expedientes de interdicción por incapacidad mental, archivos de justicia criminal, archivos de policía y registros hospitalarios, propone una aproximación a la historia de la locura basada en el análisis comprensivo de las vinculaciones entre las distintas instancias en que se debatió sobre sus características, se evaluaron sus consecuencias y se elaboraron estrategias para hacerle frente. Este enfoque revela que las alternativas existentes para sobrellevar la enfermedad mental funcionaban como respuestas temporales y flexibles que constituían una red de instancias que podían ser recorridas de distintas maneras. Sugiere que el estudio de estos itinerarios, sus actores y lenguajes resulta fundamental para comprender en toda su magnitud la forma en que fueron concebidas y enfrentadas las perturbaciones mentales en el siglo XVIII. En particular, sostiene que su funcionamiento dio pie a un debate social sobre los indicadores y significados de la locura que serviría de insumo para la sistematización del conocimiento psiquiátrico.
\end{abstract}

PALABRAS CLAVE: locura; interdicción; historia social de la locura; pensamiento médico; instituciones asilares.

\section{THE SPACES OF MADNESS IN EIGHTEENTH-CENTURY TUSCANY. STRATEGIES AND NEGOTIATIONS TO DEAL WITH MENTAL AFFLICTIONS}

ABSTRACT: This study explores the different spaces where madness was recorded in the Grand Duchy of Tuscany during the eighteenth century. Drawing from interdiction procedures, criminal records, records of the police and hospital records, it proposes an approach to the history of madness based on a comprehensive analysis of the connections between the different spaces where people debated about the characteristics and consequences of madness and developed strategies to deal with it. This analytical strategy discloses that the mechanisms for dealing with madness were temporary and flexible responses that functioned as a network that could follow different courses. The study suggests that these itineraries, its agents and languages are essential to fully grasp how mental afflictions were conceived and managed in the eighteenth century. Particularly, it argues that the way they functioned gave origin to a social debate about the indicators and signs of madness that contributed to the systematization of psychiatric knowledge.

KEY WORDS: madness; interdiction procedures; social history of madness; medical knowledge; mental institutions.

Copyright: @ 2019 CSIC. Este es un artículo de acceso abierto distribuido bajo los términos de la licencia de uso y distribución Creative Commons Reconocimiento 4.0 Internacional (CC BY 4.0). 
Durante el siglo XVIII, el encierro no era la única medida disponible para manejar la enfermedad mental y controlar sus consecuencias, y la gran mayoría de quienes eran identificados como afectos a algún tipo de perturbación mental vivían insertos en la vida social. Para los períodos en que el comportamiento de estos individuos se volvía conflictivo, las familias contaban con opciones como recluirlos en su propio hogar, el hogar de un tercero o un hospital, prisión o convento; podían pedir la ayuda de terceros y la asesoría de eclesiásticos para intentar manejar la situación sin que fuera necesario recurrir a las autoridades; o bien tomar medidas como solicitar amonestaciones policiales o la incapacitación legal. De carácter y objetivos distintos, se trataba de soluciones temporales y flexibles cuyo curso era el producto de un trabajo coordinado entre los familiares, su entorno social y las autoridades. Aunque estas opciones formaban parte del aparato administrativo del Estado, entraban en funcionamiento mayoritariamente por petición expresa de los familiares cercanos, quienes en conjunto con las autoridades debatían para encontrar una fórmula que controlara el problema, ayudara al enfermo y la familia, y asegurara la protección tanto de ellos como de la comunidad. Dado que las tipologías y posibles formas de manejar la locura no se discutían solo en los manicomios, este trabajo propone que centrarse en un solo espacio no permite comprender en toda su complejidad el fenómeno. El estudio de la locura requiere de un enfoque que abarque la multiplicidad de espacios en que se debatió, la red de estrategias para hacerle frente y las dinámicas de los itinerarios seguidos por los enfermos mentales.

La indagación de la historia de la locura ha estado ligada al problema de la reclusión y el hospital, marcada por la publicación en 1961 de Folie et Déraison. Histoire de la folie a l'âge classique de Michel Foucault (2006). Si bien sus propuestas y perspectivas analíticas siguen suscitando animados debates (Roudinesco, et al, 1996), la historiografía sobre la locura durante los últimos 30 años ha demostrado que el Gran Encierro no tuvo ni el alcance ni las características que Foucault propusiera. En cambio, se ha establecido que en Europa occidental durante la época moderna gran parte de la vida de los enfermos mentales transcurrió fuera de los muros del hospital y que, por tanto, el encierro era solo uno de los caminos posibles a seguir dentro de la variedad de estrategias utilizadas. Respecto de las nacientes instituciones asilares en particular, se ha consistentemente puesto en duda la práctica del internamiento indiscriminado, relevando que era un recurso utilizado de manera infrecuente cuya aplicación dependía de procedimientos específicos para corroborar la gravedad de la enfermedad denunciada (Porter, 1990, Bartlett y Wright, 1999, Suzuki, 1998, Suzuki, 2006, Houston, 2000, Mellyn, 2014). Todavía durante el siglo XVIII e incluso durante el siglo XIX, la locura continuó siendo una experiencia mayoritariamente doméstica.

Esta historiografía ha permitido ampliar el espectro de las formas de entender la locura en la época moderna y poner de relieve que el lenguaje para identificar y explicar las enfermedades mentales se encontraba principalmente ligado a experiencias cotidianas y saberes sociales. Se han puesto también en duda teorías respecto del abandono y trato inhumano de que supuestamente habrían sido objeto los enfermos mentales antes del mítico gesto de Philippe Pinel de liberar a los enfermos mentales de sus cadenas en el hospital de Bicêtre. Sin embargo, se han estudiado menos las conexiones entre las distintas posibilidades con que contaban las familias para enfrentar las consecuencias de la locura. De manera similar, la fascinación por el asilo aún se hace notar en publicaciones que privilegian su estudio como espacio de la locura y de la formación del pensamiento psiquiátrico por antonomasia ${ }^{1}$.

En lo que a la península itálica se refiere, Lisa Roscioni (2003 y 2011) sostiene que las primeras instituciones asilares, como Santa Maria della Pietà de Roma o Santa Dorotea de Florencia, no eran exclusivamente lugares de reclusión. Desde un principio tuvieron un propósito curativo y, por tanto, no perseguían únicamente fines de segregación, como sugirieran, entre otros, Magherini y Biotti (1992). Según Roscioni, los manicomios italianos de los siglos XVII y XVIII deben ser entendidos no solo como espacios de encierro y exclusión, sino también de cura y tratamiento de la locura, y en esa medida, como espacios formativos sustanciales para el desarrollo del alienismo desde su fundación. Esto permite datar lo que Roscioni denomina la "protoprofesionalización" del alienismo al menos dos siglos antes de la periodización tradicional, que generalmente sitúa su surgimiento a fines del siglo XVIII.

Para sustentar esta readecuación de la cronología del surgimiento del alienismo, el estudio de Roscioni posiciona su foco de estudio exclusivamente en las instituciones hospitalarias, destinando particular atención a la Pia Casa di Santa Dorotea dei Pazzerelli, 
fundada en Florencia en 1643. Examina con detención la evolución de los hospitales, la formulación del saber médico sobre la locura, el desarrollo de estrategias terapéuticas, las tipologías y significados sociales de la locura que se desprenden de los registros hospitalarios y las razones que podían llevar a que una familia solicitara la internación de un pariente. A este respecto, la historiadora establece que se recurría a la internación como último recurso y solo eran admitidas personas que desarrollaban cuadros severos que constituyeran un peligro para la sociedad o para sí mismos. Con todo, su estudio privilegia el análisis de registros hospitalarios sin profundizar en otros espacios de aparición de las enfermedades mentales, aunque ella misma es enfática en afirmar que el internamiento era solo una de las medidas tomadas por las familias para hacerles frente.

Impulsada por el interés por conocer las formas en que la sociedad Toscana se hizo cargo de la locura antes de la fundación de Santa Dorotea, Elizabeth Mellyn (2014) ha examinado el funcionamiento de estrategias para encarar las enfermedades mentales en la Toscana entre los siglos XIV y XVII. Su investigación propone que durante ese período la respuesta a la locura fue el resultado de un esfuerzo colaborativo entre las familias y oficiales de gobierno. Este orden de cosas, según la historiadora, habría encontrado su fin con la fundación de Santa Dorotea, cuya aparición generó un punto de inflexión al ofrecer una solución definitiva al problema del destino de los enfermos mentales.

Esta cronología, y el lugar predominante que se le otorga a Santa Dorotea, desconoce que el manejo de la enfermedad mental no se reduce a su institucionalización y que el modelo colaborativo durante todo el siglo XVIII continuó siendo la tónica que primó como respuesta. Por un lado, no obstante reconocer que la profesión médica no ejerció el predominio en las formas de entender y actuar frente a la locura entre los siglos XIV y XVII, al asumir que la fundación de Santa Dorotea resolvió el problema del destino de los enfermos mentales, esta historiografía sigue atribuyéndole un rol preponderante a la institucionalización y la medicina (Magherini y Biotti,1998, Roscioni, 2003, Mellyn, 2014, Conti 2015). Por otro lado, centrarse en el manicomio no permite abarcar la dimensión extrahospitalaria y la riqueza de saberes no médicos existentes sobre la locura. Cuando se amplía el marco de análisis fuera de la práctica y la discusión médica, la configuración del conocimiento alienista se perfila como la culminación de una larga discusión social respecto de sus características, sintomatología y tratamiento.

En atención a esto, este trabajo ofrece una aproximación a la historia de la locura que examina las lógicas y estrategias tras las diferentes respuestas a la locura de manera conjunta e interrelacionada. A través del estudio de expedientes de interdicción por incapacidad mental, archivos de policía, archivos de justicia criminal y registros hospitalarios del Gran Ducado de Toscana en el siglo XVIII, revisados en mi tesis doctoral (Labarca, 2015), planteo que abordar las concepciones y respuestas a la enfermedad mental desde este enfoque posibilita observar aspectos que de otra manera permanecerían ocultos. En particular, revela que la enfermedad mental se entendió como un fenómeno del cual la comunidad en su conjunto era responsable, y que la red de alternativas y los itinerarios seguidos por quienes supuestamente sufrían la enfermedad dieron pie a un debate entre los actores sobre los indicadores y significados de la locura que serviría de insumo para la sistematización del conocimiento médico y legal ${ }^{2}$. A este respecto, propongo que las diferentes alternativas disponibles para ayudar a las familias a enfrentar las consecuencias económicas, familiares, emocionales y sociales de la locura funcionaban como una red interconectada, no eran ni excluyentes ni correlativas y conformaban una trama que se articulaba en base a cada historia personal y a las condiciones familiares concretas en que la enfermedad hiciera su aparición. Para dar cuenta de esto, examino las distintas instancias de reclusión, las dinámicas de internación en Santa Dorotea y su impacto en el funcionamiento del entramado de respuestas, y el funcionamiento de los procesos de interdicción, para luego reflexionar sobre la configuración de los itinerarios de la locura y sus potencialidades para el estudio de las enfermedades mentales en el pasado.

\section{RECLUIR}

Dentro del abanico de alternativas disponibles para enfrentar los problemas ocasionados por la enfermedad mental durante la época moderna, el mecanismo que sin duda más ha concentrado la atención de la historiografía es el encierro, y específicamente, la internación en manicomios o casas de locos. Sin embargo, la historia del cuidado y manejo de la locura en la Toscana moderna no se reduce a la labor de 
Santa Dorotea. Por ejemplo, las familias podían solicitar el encierro temporal en una prisión o una fortaleza, como Le Stinche y la Fortezza da Basso, ambas ubicadas en Florencia. Asimismo, se podía solicitar el confinamiento en la isla de Giglio, en Volterra o en cualquiera de los territorios usualmente utilizados por el sistema judicial para la pena de confinamiento. Estas peticiones de encierro eran centralizadas por los tribunales criminales, organismos estatales encargados del orden público y la policía, que las cursaban muchas veces recurriendo al ejercicio de su poder ejecutivo ${ }^{3}$.

Esta dinámica llevaría a los primeros estudiosos de la locura en la Toscana moderna a aseverar que a partir del siglo XVI la cárcel y la galera se convirtieron en un recurso frecuente utilizado por las autoridades para solucionar los problemas ocasionados por criminales, pobres y locos en forma indiscriminada (Magherini y Biotti, 1992 y 1998, Biotti, 2002). Aunque reconocen que los principales promotores del encierro eran las familias, Magherini y Biotti sostienen que durante este siglo el poder de las familias se vio progresivamente mermado por el creciente poder estatal. Sin embargo, estudios recientes han revisado los alcances del recurso del encierro para encarar el problema de la locura en la Toscana moderna. En esta línea, Mellyn (2014) argumenta que el proceso de centralización y fortalecimiento del Estado emprendido por la administración de los Medici (siglos XVIXVII) no significó una época negra para la enfermedad mental, toda vez que no se tradujo en un aumento del número de encarcelados ni significó un recrudecimiento de medidas coercitivas. Frente a este panorama, la historiadora asevera que durante estos dos siglos las instituciones públicas ejercieron un rol limitado en la gestión de la enfermedad mental y que el destino principal del enfermo mental durante el período fue el espacio doméstico.

Ahora bien, los archivos de policía y de justicia civil y criminal que he podido revisar (Labarca, 2015) ponen de manifiesto que este estado de cosas continuó siendo la norma luego de la fundación de Santa Dorotea y que durante el siglo XVIII el destino de los enfermos mentales continuaría sujeto a las posibilidades de las familias y su red de apoyo. Las personas recurrían a la intervención del engranaje administrativo del Estado granducal solo cuando esta red fallaba, siendo generalmente un recurso de última instancia. Las peticiones de intervención ocurrían una vez que los intentos domésticos por controlar los efectos per- niciosos de la conducta disruptiva habían resultado en vano. Se recurría primero a la vecindad y a los párrocos, y luego a las autoridades, solicitando en primera instancia que convencieran, amonestaran o amenazaran a la persona para que cambiara o controlara su conducta, y solo después a medidas más drásticas como la reclusión o la interdicción. La documentación que he examinado sugiere que el encierro no era el ideal ni para las familias ni para las autoridades, pues significaba un gasto que era preferible evitar de no ser estrictamente necesario, especialmente para las familias más pobres, dado que todo recluso debía financiar su mantención. Para las familias más privilegiadas, recluirlos en residencias separadas, preferentemente lejos de la familia, o pagar a terceros para que los recibieran en las suyas eran alternativas preferibles. El confinamiento en territorios apartados, en cambio, se erigía como una alternativa mejor para las familias desposeídas, toda vez que el sustento del confinado dependía de su propio trabajo 4 .

Los casos que llegaban a manos de la administración, ya fuera por solicitud de familiares o porque habían sido derivados por la justicia criminal, daban inicio a una verdadera negociación entre familiares y autoridades. Esta negociación, más que establecer si se justificaba el encierro, se focalizaba en definir sobre quién recaía la responsabilidad del cuidado, qué implicaba, y cómo se podía salvaguardar el orden público y a la vez proteger el honor y patrimonio de las familias. Así, el destino de los criminales considerados inimputables por causa de una afección mental, o la decisión de qué hacer ante las solicitudes de intervención para controlar el desorden ocasionado por quienes eran percibidos como irracionales o enfermos mentales, se resolvía de manera casuística. En este contexto, son numerosos los casos en que las autoridades se veían enfrentadas al dilema de una familia que no contaba con los recursos para sostener a un pariente que exigía cuidados especiales o que dada su supuesta peligrosidad constituía un peligro para la sociedad.

Una alternativa distinta a la prisión y el confinamiento era embarcar a la persona como tripulante de navíos de cualquier nacionalidad y rubro. Esta opción era utilizada exclusivamente con hombres jóvenes cuyo comportamiento, descrito de forma muy similar a como era descrita la perturbación mental en otros espacios, escapaba a los márgenes de lo aceptable. Como se observa en numerosos comenta- 
rios de los oficiales de gobierno encargados de tramitar estas peticiones, se pensaba que enrolar a estos jóvenes "díscolos" como tripulantes podía ejercer un efecto reformador que aquietara el "cerebro extravagante", "carácter irregular" y "ánimo difícil, perturbado e inquieto" que los gobernaba ${ }^{5}$.

Por último, dentro de las opciones de encierro existentes, las familias más pudientes podían destinar a quienes sufrían de una afección mental que les resultaba problemática a una vida conventual. El convento podía resultar una alternativa útil para proveer de los cuidados necesarios a una viuda anciana que presentara problemas de salud física y mental cuyos hijos rehusaban asumir ${ }^{6}$. Es posible encontrar también registros de mujeres catalogadas como víctimas de algún tipo de perturbación mental que habían sido enviadas a monasterios, a veces como medida transitoria para alejarlas de un pretendiente no deseado por el resto de la familia o para ocultar algún desorden de comportamiento ${ }^{7}$. De manera similar, los registros documentan que el convento era una alternativa para hombres de bien luego que una serie de encarcelamientos y arrestos domiciliarios habían probado ser inútiles para poner freno a las consecuencias de sus continuas "frenesías" y "demencia". Ahora bien, aunque es difícil establecer hasta qué punto las familias recurrían al monasterio, existen indicios que matizan su alcance. Se pueden encontrar numerosos registros de personas enviadas desde un monasterio a Santa Dorotea o bien de vuelta a sus familias porque su perturbación mental interrumpía el desarrollo de la vida monástica9 ${ }^{9}$ Las fuentes también sugieren que se consideraba que el monasterio en sí podía desencadenar alteración anímica y perturbación mental con cuadros de delirio melancólico, de comportamiento suicida o accesos de furia ${ }^{10}$.

\section{LA FUNDACIÓN DE SANTA DOROTEA}

Fundado en 1643, el hospital de Santa Dorotea abrió sus puertas por primera vez en 1647, definiéndose como una institución dedicada exclusivamente al cuidado de "quienes no fueran de mente sana, llamados vulgarmente locos [pazzi]"11. Desde su inauguración los internos estuvieron bajo el cuidado de un cirujano residente y un médico de visita encargado del tratamiento y cura de los enfermos ${ }^{12}$, y a partir de 1756 el hospital contó con médico permanente y dos médicos de consulta ${ }^{13}$. El hecho que los pacientes de Santa Dorotea se encontraran bajo el cuidado mé- dico desde un principio, aunque contara con médico permanente solo desde mediados del siglo XVIII, permite sostener que la institución desde sus inicios concibió la locura como una enfermedad que podía ser tratada y curada (Roscioni, 2003).

Desde su fundación el hospital Santa Dorotea enfrentó problemas de financiamiento que se tradujeron en periódicas crisis económicas que, sumadas a desastres naturales como los provocados por las salidas de cauce del Arno, pusieron en repetidas ocasiones en peligro la continuidad del establecimiento. La dificultad de encontrar suficientes donaciones explica por qué sus estatutos establecieron que solo podían ser recibidos aquellos enfermos que pudieran financiar su estadía. Además de pagar la cuota requerida por la congregación, cada paciente debía además encargarse de su alimentación y de proveerse de enseres básicos como cama, ropa y sábanas ${ }^{14}$. Todo el resto del funcionamiento del hospital dependía de la caridad, la que no fue suficiente para financiar el gasto que implicaba mantener el edificio, pagar el personal, el cirujano residente, el médico de visita y los servicios básicos de cada enfermo.

Para albergar al segmento de población excluido de Santa Dorotea, en 1688 se creó una sección especial para enfermos mentales dentro del hospital general del Gran Ducado de Toscana, Santa María Nuova, pero el pequeño tamaño de esta pazzeria no resultó suficiente para solucionar el problema de los enfermos desposeídos. Mientras los registros de esta última son esporádicos, los archivos de Santa Dorotea documentan 429 admisiones entre 1647 y 1750, lo que da una media de 15 pacientes al año (Roscioni, 2003, p.117) $)^{15}$. Esto explica que en 1720 el director del Hospital Santa María Nuova declarara que Santa Dorotea debía ser cerrado para evitar que continuara consumiendo los escasos recursos públicos existentes, argumentando que la institución albergaba solamente a los enfermos que podían pagar su mantención, es decir, justamente aquellos que poseían los medios para ser cuidados en sus casas y que no necesitaban de la caridad. En cambio, señalaba el funcionario, los enfermos mentales "más necesitados" eran rechazados y en algunos casos obligados a vagar sin ningún cuidado, constituyendo un peligro para ellos y la sociedad (Roscioni, 2003, p. 87).

Santa Dorotea continuaría funcionando de la misma manera hasta que en 1750 la Regencia promulgó una reforma que lo transformó en Hospital Gran Ducal para "curar todas las enfermedades comprendidas en 
la manía y para custodiar a aquellas que son incurables"16. La reforma también estableció la gratuidad para todos aquellos locos furiosos (pazzi furiosi) cuya pobreza fuera demostrable, lo que permitió que la media de internos por año subiera considerablemente, llegando a albergar un promedio de 65 enfermos al año entre 1750 y 1788, con una clara progresión que va desde 16 pacientes por año en 1751 a 134 en 1785. Las estadías eran cortas y los reingresos no eran raros: del total de 2411 ingresos del período, $446(18,5 \%)$ corresponden a pacientes que fueron internados repetidas veces y alrededor de la mitad estuvieron menos de tres meses internados (Roscioni, 2003, pp. 138-139).

A pesar de estos números, existen varios indicios que permiten matizar el alcance de la fundación de esta institución, tanto en términos de su impacto en el destino de los enfermos mentales, como en términos de su representatividad respecto de cómo era entendida y manejada la locura en el siglo XVIII. Aunque se observa un notorio aumento en las cifras de ingreso, el contraste con la documentación que he podido revisar proveniente de otras instancias judiciales y privadas donde quedó registrada la locura sugiere que el hospital no reemplazó a las anteriores formas de manejar la enfermedad mental (Labarca, 2015). En primer lugar, me parece fundamental tener presente que la internación duraba generalmente un período breve, siendo en escasísimas ocasiones de por vida. En segundo lugar, cualquier análisis sobre el impacto y alcance reales de esta institución debe tener en cuenta que las instituciones asilares solo admitían cuadros agudos, por lo que no constituyen una muestra representativa de las tipologías de enfermedad mental concebidas en la época, ni abarcan todo el abanico de síntomas y signos que la componían.

La pazzia furiosa era considerada un estadio puntual dentro del curso de una enfermedad mental que podía mitigarse, sanar, o bien manifestarse episódicamente, como sugiere la corta duración tanto de las internaciones como de la incapacitación legal (interdicciones). Por lo demás, al examinar otros espacios en que la enfermedad mental fue discutida queda de manifiesto que tanto médicos como familiares y autoridades concebían y distinguían distintos tipos de enfermedad mental según síntomas, grado de compromiso cognitivo y anímico e impacto en la vida de los enfermos y de su entorno. Por ejemplo, en 1756 una mujer fue internada a petición del marido como pazza furiosa, lo que a juicio de las autoridades quedaba probado porque había intentado defenestrar a sus hijos y luego había interrumpido una misa casi desnuda con gran escándalo de los feligreses ${ }^{17}$. Ahora bien, esta caracterización no puede ser tomada como modelo de cómo era representada la locura ni como la única forma bajo la cual los contemporáneos pensaban que esta se manifestaba.

Si bien síntomas típicos de la internación como la conducta suicida, el comportamiento "frenético", la manía, las fijaciones de mente o el delirio melancólico aparecen también en caracterizaciones de la locura fuera del contexto de Santa Dorotea, no son los más frecuentes. En cambio, para el contexto hospitalario constituían síntomas imprescindibles para justificar el ingreso. Santa Dorotea solo admitía enfermos diagnosticados con locura furiosa (pazzia furiosa), definida como aquella que constituía un peligro para la propia vida, su entorno familiar y la comunidad ${ }^{18}$. Esto en la práctica comprendía a quienes cometían intentos de suicidio, amenazaban la vida de sus más cercanos, o alteraban el orden público y la moral. A esto se suma que hasta 1750 la internación debía ser financiada por los familiares, por lo que los pacientes tendían a provenir exclusivamente de sectores privilegiados. Si bien a partir de 1750 se establecieron mecanismos de financiación pública, estos estaban estrictamente limitados a casos probadamente peligrosos de enfermos cuyas familias carecían de redes o medios para poder cuidarlos, como fuera el caso de la mujer arriba citada.

Dado que la internación por cargo público constituía un peso para la comunidad, la evolución de estos casos era constantemente revisada para decretar su egreso lo antes posible. Para este efecto, el médico de cabecera del hospital se encontraba en permanente comunicación con las autoridades florentinas para informar sobre el estado de los pacientes e identificar quiénes podían salir del hospital. Cuando no se podían encontrar familiares que se responsabilizaran del paciente una vez reintegrado en sociedad, se hacía necesario encontrar soluciones alternativas que suponían una negociación entre el jefe de policía y las autoridades locales. Esta negociación resulta interesante, pues demuestra que el hecho que el hospital solo admitiera enfermos por períodos breves no significa que estos no contaran con el apoyo de la comunidad.

La internación durante todo el siglo siguió siendo utilizada por las familias como último recurso, incluso luego que se estableciera la gratuidad para los que 
no pudieran financiar el cobro exigido por el hospital. En consecuencia, la enfermedad mental aparece en los archivos solo por circunstancias excepcionales que no pueden ser tomadas como las circunstancias "normales" de la "anormalidad", sino solo como las contingencias que explican su registro. Éstas, como ha señalado Robert Allan Houston en su estudio sobre la locura en la Escocia del siglo XVIII, no estaban necesariamente vinculadas ni condicionadas a un cambio en el curso de la enfermedad mental denunciada, y en muchos casos incluso no tenían ninguna relación (Houston, 2000, p. 173). Por ejemplo, son comunes las peticiones de internación que señalaban que la persona sufría del cuadro de pazzia furiosa desde hacía años, o que tenía una tendencia, sin especificar si era reciente o no, a manifestar la conducta propia de ese tipo de enfermedad.

\section{LA INTERDICCIÓN Y EL ROL DEL MAGISTRATO DEI PUPILLI ET ADULTI}

Una institución que resulta fundamental para estudiar las concepciones y el manejo de las enfermedades mentales a lo largo de toda la época moderna en el Gran Ducado de Toscana es el Magistrato dei Pupilli et Adulti (Labarca, 2015). Esta magistratura, fundada a fines del siglo XIV y disuelta en 1808, fue creada para hacerse cargo de la tutela de menores, pero a partir de 1473 empezó a ocuparse de la curatela de adultos "mentecatos [mentecapti] o fuera del natural sentimiento [fuori del naturale sentimento]", lo que comprendía tanto a quienes no se encontraban en posesión de sus facultades mentales como a sordomudos $^{19}$. En 1718 el Magistrato dei Pupilli adquirió jurisdicción exclusiva sobre la curatela de adultos, que ahora podía decretarse para "mudos y sordos de nacimiento, furiosos, mentecatos, dementes, pródigos, dilapidadores u otros a los cuales es conveniente que les sea interdicta la administración de sus haberes y facultades" 20 .

Según los estatutos, la interdicción se podía solicitar por cualquiera de las causales antes citadas, pero en la práctica, las categorías que se utilizaron fueron la sordomudez, la demencia y la prodigalidad. Estas dos últimas no se encontraban realmente diferenciadas, funcionando como categorías intercambiables. La demencia era una denominación genérica utilizada para categorizar a todos los tipos de enfermedad mental, desde los congénitos a todo el espectro de perturbaciones mentales, independientemente del compromi- so de las facultades cognitivas. Por su parte, la prodigalidad hacía referencia en principio a la disipación desmedida del patrimonio, pero como el comportamiento de quien la realizaba era considerado en la Toscana como un tipo de incapacidad que debía atribuirse a una perturbación mental, terminaba entremezclándose con la demencia hasta resultar, en la práctica, equivalentes (Labarca, 2015).

Las interdicciones se iniciaban con el envío de una súplica suscrita por familiares cercanos donde debían fundamentar por qué consideraban necesaria la medida y probar sus declaraciones con al menos dos testimonios. Los testigos eran en su mayoría miembros del clero, conocidos, vecinos o notables de la localidad, y en menor medida, médicos o cirujanos -en solo 50 de un total de 600 interdicciones que he podido contabilizar entre 1700 y 1775 se recurrió a la opinión médica para certificar la demencia. A partir de estas peticiones, los magistrados iniciaban una investigación solicitando información a las autoridades de la localidad de origen del demandado, o corroborando a través de visitas directas a su domicilio. Estas diligencias buscaban establecer si la denuncia tenía fundamentos, qué consecuencias acarreaba para la persona y su entorno, cuál era la situación económica de la familia y cómo era la relación entre sus miembros. Por ello, las peticiones sumaban a la denuncia de demencia o prodigalidad una serie de descriptores para dar cuenta de la tipología de la supuesta incapacidad y sus consecuencias económicas y relacionales. Todas estas consideraciones eran tomadas en cuenta para decretar la interdicción, que privaba a la persona de su derecho a administrar sus bienes y la dejaba bajo la curaduría de la magistratura, que nombraba a un "actor" para administrar el patrimonio y representar a la persona interdicta ante la ley.

Dado que el universo de desórdenes mentales que podían caber dentro de las categorías de demencia y prodigalidad eran amplísimos -justamente porque eran categorías inespecíficas-, los expedientes de interdicción entregan un amplio registro de lo que se entendía por enfermedad mental en el siglo XVIII. En la medida que la petición de interdicción no requería convencer a las autoridades de que la enfermedad identificada en la persona justificaba una internación -es decir, que su conducta constituía un peligro para sí o para la sociedad-, las denuncias y posteriores investigaciones daban cabida para retratar distintos grados de compromiso cognitivo y trastorno afectivo. 
Aunque el paso inicial de un proceso de interdicción era la constatación de que el desorden mental denunciado efectivamente incapacitaba a la persona para administrar sus bienes, en muchos casos la denuncia operaba como una puerta de entrada para discutir los signos de la enfermedad mental más allá del comportamiento financiero o de su peligrosidad. Familiares, autoridades y testigos sopesaban, por ejemplo, las características y significados de indicadores como la inquietud, la inestabilidad anímica, el actuar extravagante, la angustia, la sensación de opresión, la irascibilidad, la venta o el gasto compulsivos, la impulsividad, el desgano y la renuencia al trabajo.

Otra característica específica de la labor del Magistrato dei Pupilli et Adulti es que supervisaba la curatela mientras durara la interdicción, fiscalizando no solo la correcta administración del patrimonio del interdicto, sino realizando también un riguroso seguimiento de todos los asuntos privados que lo involucraran para resguardar sus intereses y los de su familia, que no siempre coincidían. Como consecuencia, para el siglo XVIII contamos no solo con los expedientes de los procesos de interdicción, sino también con una serie de documentos anexos que permiten realizar un seguimiento de estas familias durante los años de la curatela ${ }^{21}$. Más aún, dado que este procedimiento podía solicitarse y revocarse cuantas veces los parientes y las autoridades lo consideraran necesario, es posible reconstruir distintas facetas de la locura en todas sus expresiones, en muchos casos durante toda la vida adulta de una persona. Por ejemplo, he podido seguir el derrotero de Ranieri Navarretti, que aparece en 1717 internado en Santa Dorotea y reaparece en los registros del Magistrato dei Pupilli interdicto en 1727 como demente, donde continúa apareciendo sin interrupciones hasta su muerte en 1757, todavía bajo curatela. Algo similar ocurre con el caso de Angiolo Barchesi, interdicto 5 veces entre 1735 y 1754 e internado repetidas veces en la pazzeria de Santa María Nuova, siempre por períodos breves ${ }^{22}$.

Por ello, la documentación del Magistrato dei Pupilli constituye una fuente ineludible para el estudio de la locura en el Gran Ducado de Toscana que, al complementarse con la red de alternativas para hacer frente a sus consecuencias, complejiza la visión que se obtiene al estudiar los registros hospitalarios por sí solos. En particular porque la documentación de las nacientes instituciones asilares, a diferencia de los expedientes de interdicción y curatela, solo registran la enfermedad en un momento dado. Estas descripciones no incorporan el devenir de la enfermedad, pues siendo fotografías del momento de la internación, no permiten saber sobre el contexto del enfermo, ni del curso anterior o posterior de la enfermedad. Los archivos de Santa Dorotea no cuentan, por lo demás, con registros del tratamiento ni de la evolución de la enfermedad durante la internación, y la documentación de los ingresos y egresos es en general muy escueta, y no da cuenta de las concepciones de quienes denunciaban o dirimían cada caso. Por último, resulta particularmente relevante notar que la mayoría de los interdictos no eran internados ni antes, ni durante, ni después de la interdicción -solo 14 de las 600 interdicciones registran una conexión con Santa Dorotea o la pazzeria de Santa María Nuova-. Este hecho demuestra que en el siglo XVIII el hospital no constituía un destino obligado de los enfermos mentales y que las estrategias para ayudarlos, lidiar con ellos y con las consecuencias de su enfermedad eran variadas.

En un principio el espacio de intervención del Magistrato dei Pupilli estaba reducido predominantemente a familias que poseyeran un patrimonio suficiente como para poder solventar el gasto que significaba declarar incapaz a un familiar, lo que lo hacía un recurso casi exclusivo para las familias más ricas. Sin embargo, a partir de 1767 se garantizó el acceso a la interdicción de manera gratuita para "miserables y pobres" que no pudieran financiar los costos del proceso ni la tarifa cobrada por la magistratura por la supervisión de la curatela y la administración del patrimonio. Este cambio produjo un notable incremento en el número de interdicciones, ampliando considerablemente la muestra de tipologías de enfermedad mental a la que podemos acceder.

Según los registros examinados por Mellyn (2014), entre el siglo XIV y mediados del XVII las curatelas constituyen algo más de la mitad de los 300 casos de locura que logró identificar en los archivos de justicia criminal y civil toscanos. Estas cifras contrastan con el alto número de interdicciones decretadas durante el siglo XVIII. Solo entre 1700 y 1775 he podido registrar 600 interdicciones en los archivos del Magistrato dei Pupilli. Como se puede observar en la figura 1, el número de interdicciones creció de manera constante a lo largo del período, incremento que demuestra que las familias recurrieron con creciente frecuencia a las instituciones públicas para negociar estrategias y respuestas a los problemas de la locura. 
Figura 1. Número de interdicciones administradas por el Magistrato dei Pupilli entre 1700 y 1775 (cada 5 años)

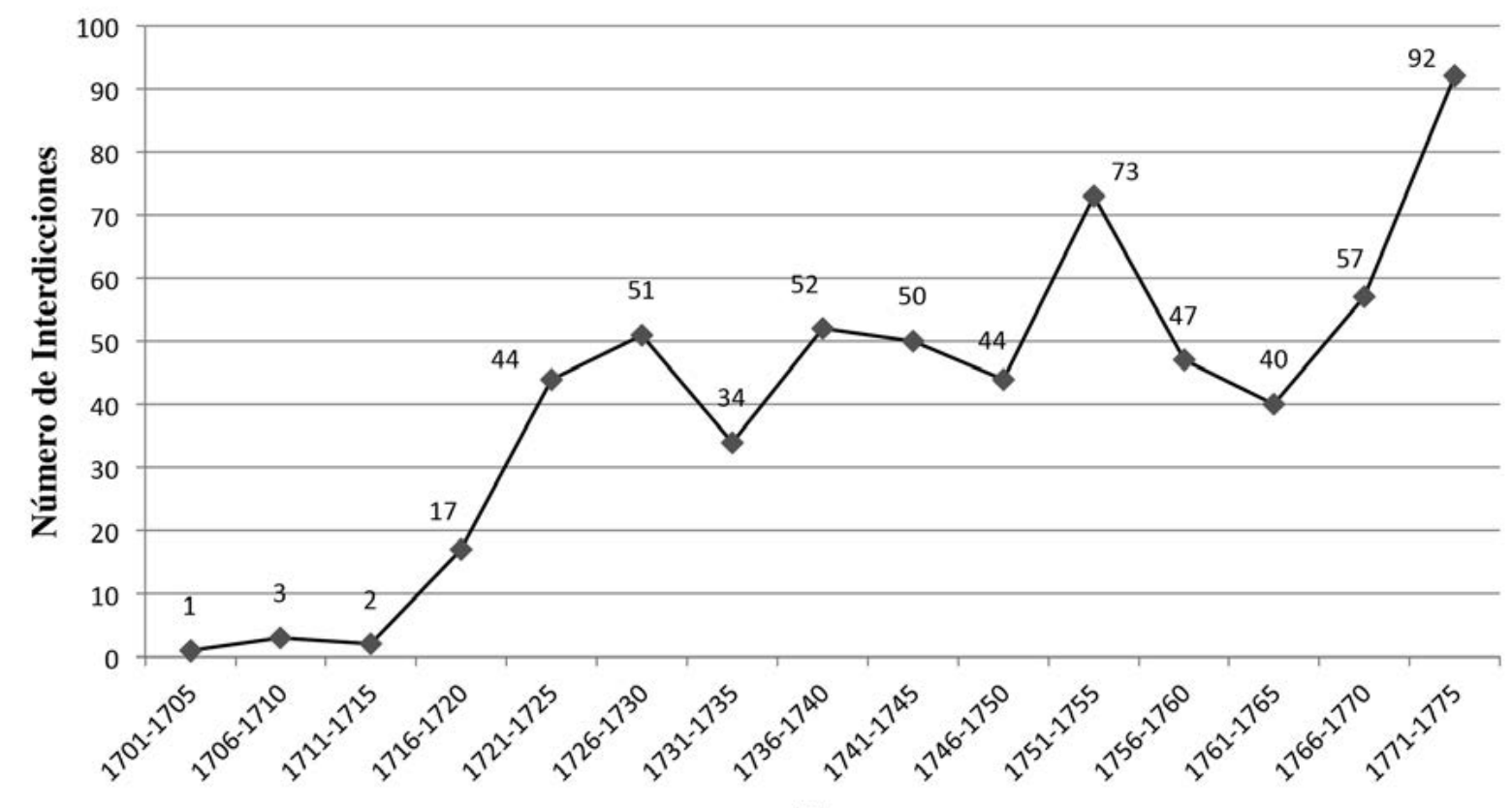

Años

Fuente: He recabado estos datos de ASF, MPAP, Memoriali e Negozi di Cancelleria, F. 2299 - F. 2310 y Campione di Deliberazioni, e Partiti, F. 112 - F. 170.

Frente al abanico de alternativas disponibles para manejar la locura en el siglo XVIII, la interdicción aparecía como un camino que podía ser tomado paralelamente a los otros y que involucraba además una dimensión familiar fundamental. Mientras opciones como el encierro o la amonestación policial perseguían propósitos curativos o coercitivos, respectivamente, la interdicción tenía una doble función. En primer lugar, permitía evitar o minimizar las consecuencias económicas de la enfermedad mental y, en segundo lugar, introducía un árbitro para dirimir conflictos entre el interdicto y sus familiares y para elaborar caminos de salida que conciliaran los intereses de cada una de las partes involucradas. Por ejemplo, dentro de las facultades de los oficiales de la magistratura encontramos determinar dónde debía vivir el interdicto, quién debía hacerse cargo de su cuidado, cuánto dinero debía destinarse a su manutención y la de su familia, autorizar uniones matrimoniales o bien determinar si ameritaba decretar la separación temporal de un matrimonio cuando la perturbación de uno de los cónyuges hacía imposible que la convivencia se pudiera llevar de manera pacífica. Intervenían también para defender los intereses de enfermos mentales que según su propia denuncia o según testigos no eran bien tratados por sus familiares o bien eran víctimas de sus estrategias malintencionadas con miras a usurpar sus bienes aprovechándose de su estado de debilidad.

\section{EL ENTRAMADO DE RESPUESTAS A LA ENFERMEDAD MENTAL}

La historiografía sobre la locura concuerda en sostener que durante el siglo XVIII las enfermedades mentales alcanzaron una visibilidad inédita (Porter, 1990, Suzuki, 1991 y 1992, Roscioni, 2003, Rabin, 2004, Rushton, 1988, Labarca, 2015, entre otros). Para el caso de la Toscana, ello se observa en primera instancia en el incremento de registros de la locura en distintas instituciones públicas: aumentan los ingresos en las instituciones asilares, los procesos judiciales en que se debate la responsabilidad criminal del enfermo mental y las peticiones de interdicción. También se hacen más frecuentes las peticiones de familiares que solicitaban reclusión, mediación o amonestación de personas que eran definidas como víctimas de una perturbación, y los casos manejados por la policía. Más que sugerir un incremento en la prevalencia de enfermedades mentales, el hecho que la locura aparezca registrada en diversos espacios institucionales 
con mayor frecuencia que en los siglos anteriores revela, a mi juicio, que las familias toscanas recurrieron a las instancias públicas con mayor frecuencia que en los siglos anteriores para encarar el impacto que esta enfermedad infligía en la vida familiar y en la gestión patrimonial. Así, el modelo colaborativo observado para los siglos anteriores (Mellyn, 2014) se puede aplicar también para el siglo XVIII, con la diferencia que las familias recurrían a las autoridades con mayor frecuencia que en los siglos anteriores. A la vez, esta dinámica sugiere que la enfermedad mental no se concebía como un asunto privado sino social, que requería de soluciones colectivas para garantizar la tranquilidad de todos y asegurar el bienestar del enfermo. Aunque coordinadas por los oficiales de gobierno, las estrategias eran diseñadas con la activa participación de la sociedad.

Como se expresó más arriba, las peticiones en muchos casos no solo buscaban la interdicción o el encierro, sino que apuntaban sobre todo a resolver conflictos, buscar medios de combatir la enfermedad, distribuir responsabilidades y proponer alternativas cuando ninguno de los miembros de la familia estaba dispuesto a cohabitar con el enfermo o cuando por razones económicas no podían hacerlo. Se ha hablado de los abusos y estado de indefensión en que se encontraban los enfermos mentales en el pasado (Stagnaro, 2015), pero se ha discutido menos sobre las instancias de protección que se evidencian en las fuentes que he examinado acá. El ejemplo de la interdicción es sintomático en este sentido: funcionaba como una instancia para garantizar el cuidado de estos enfermos que, aunque significaba revocar su personalidad jurídica, permitía a la vez defender otros derechos fundamentales. Más aún, cuando se combinan estos registros con la documentación dejada por los otros espacios de la locura, las formas en que era comprendida y manejada cobran nuevos sentidos, complejizando la visión tradicional sobre la estigmatización e indefensión de los enfermos mentales.

Por un lado, la interdicción suponía todo lo contrario al ocultamiento de la condición mental del familiar, en la medida que se trataba de un proceso que no solo involucraba a testigos y autoridades, sino que también conllevaba de por sí la publicidad de la condición denunciada. Todo decreto de interdicción debía ser informado públicamente a la comunidad para notificar que la persona había quedado judicialmente incapacitada de participar en actividades económicas - despojar a una persona de su capacidad jurídica su- ponía que cualquier contrato u obligación contraída luego de la interdicción era automáticamente inválida-. Así, el alto número de interdicciones que se registran en el período sugiere que las familias, en especial las acomodadas, no buscaban esconder los desórdenes mentales.

Por otro lado, aproximarse a los espacios entendiéndolos como una red interconectada de recursos para enfrentar los desafíos y dificultades que ocasionaban quienes eran definidos como víctimas de enfermedad mental permite observar la variedad de visiones y respuestas que había sobre el problema. $\mathrm{Si}$ bien es posible encontrar referencias a enfermos que permanecían recluidos en residencias familiares, a veces encerrados en una habitación, la mayoría de las veces quienes eran objeto de una interdicción o eran internados en un hospital llevaban una vida inserta en la sociedad: eran casados, tenían hijos, manejaban sus asuntos financieros de manera activa y desempeñaban actividades laborales regulares. De manera similar, prácticamente no es posible encontrar indicios del trato inhumano de que supuestamente habrían sido objeto los enfermos mentales antes del surgimiento del trato humanizado propugnado por médicos como Vincenzo Chiarugi, Philippe Pinel o William Tuke. No solamente las referencias a enfermos encadenados en los manicomios o en sus casas son extremadamente escasas, sino que también se registran abundantes expresiones de empatía, afecto y cuidado por parte tanto de los familiares como de las autoridades.

Todas las medidas disponibles para manejar las enfermedades mentales en el Gran Ducado de Toscana deben ser entendidas como una red interconectada de alternativas a las que recurrir para hacer frente a los problemas que generaba la locura. Dada su diversidad, la elección de una u otra dependía en gran medida de la forma en que la conducta disruptiva incidiera en la vida social y familiar. Por ejemplo, si la preocupación se fundaba en la peligrosidad del enfermo, lo más lógico era recurrir al hospital o a la ayuda de terceros que se pudieran hacer cargo. Sin embargo, esta necesidad podía ser frenada por impedimentos como la política de admisión del hospital, la capacidad de la familia para solventar el gasto que una u otra alternativa significaba, la composición del grupo familiar y sus conflictos, y las características del desorden mental denunciado.

Como se dijo arriba, Santa Dorotea solo recibía enfermos cuyo estado mental supusiera un peligro para 
ellos o para la sociedad. Si no se probaba la conducta suicida o peligrosa para la integridad de otros, si sus brotes maníacos eran esporádicos sin constituir un patrón o un estado permanente, o si, en suma, se determinaba que la necesidad de enviarlos al hospital surgía no de la imposibilidad de controlarlos y protegerlos, sino de la dificultad de cuidarlos (porque no se querían hacer cargo de esa responsabilidad, porque no contaban con los medios o la infraestructura, porque no tenían quién lo hiciera), el hospital no era una opción. Más aún, dado que duraba en la mayoría de los casos entre uno y tres meses, la internación no resolvía el conflicto.

En otras ocasiones la preocupación podía ser su conducta desafiante, escandalosa y atentatoria contra el honor y decoro de la familia, en cuyo caso la opción de una admonición del jefe de policía o la prisión como correctivos eran los más utilizados. Finalmente, cuando el objetivo de recurrir a la justicia era frenar la disipación del patrimonio, la interdicción aparecía como la mejor opción. Solicitar un curador servía, además, para manejar los conflictos relacionales propios de la convivencia con las alteraciones anímicas de la perturbación mental.

Las alternativas existentes para sobrellevar la enfermedad mental funcionaban de manera paralela, se encontraban desvinculadas entre sí, eran temporales y flexibles. En este entramado, la labor ejercida por el Auditore Fiscale en su calidad de jefe de policía y miembro del Consejo de Regencia funcionaba como vértice articulador. Más aún, es la única autoridad que aparece registrada en todos los espacios institucionales y legales ocupados del manejo de la locura: coordinaba los ingresos en Santa Dorotea, se encargaba de arrestos y/o amonestaciones a petición de familiares para llamar al orden a los individuos problemáticos, corroboraba decisiones de jueces respecto de casos de locura en la justicia criminal. Por último, colaboraba también con la labor del Magistrato dei Pupilli interviniendo cuando se decretaban reclusiones domiciliarias o cuando se requería lograr acuerdos relativos a alojamiento, visitas domiciliarias para supervisar situaciones de vida o intervención in situ en conflictos familiares. Su intervención podía ser solicitada directamente por las familias, como es el caso de una mujer que pidió sucesivas medidas para hacer frente a la violencia y maltratos de que era víctima por parte de su marido interdicto por incapacidad mental: primero reclusión en una villa rural lejos de la vivienda principal en Pescia, luego una orden de alejamiento y por último su reclusión en una Fortezza ${ }^{23}$. El Auditore Fiscale hablaba con los familiares y con los supuestos enfermos mentales, intercedía entre ellos, pesquisaba opiniones en la comunidad, se comunicaba con las autoridades locales y vecinales, miembros del clero, profesionales médicos si es que los había o si es que estaban involucrados, y con oficiales de otras magistraturas.

\section{LOS ITINERARIOS DE LA LOCURA COMO HERRAMIENTA METODOLÓGICA}

Los espacios de la locura en la Toscana del siglo XVIII constituían una red de instancias que podían ser recorridas de distintas maneras, trazando caminos que dependían de las circunstancias particulares que rodearan a cada caso y el tipo de perturbación identificada. Los itinerarios seguidos por quienes eran identificados como enfermos mentales confluían en la labor del Magistrato dei Pupilli y del Auditore Fiscale, que funcionaron como coordinadores de sus dinámicas. Medidas como recurrir a la ayuda de terceros, solicitar la intervención de autoridades, encerrar en prisiones, confinar o embarcar, enviar a un convento, internar en un hospital o solicitar la interdicción eran opciones que se podían tomar de manera sucesiva, paralela o aislada. Su puesta en práctica supuso la negociación y requirió de la colaboración entre autoridades, jueces, familiares $y$, en menor medida, médicos, para generar nuevas respuestas al problema de la locura y encontrar un equilibrio entre los intereses públicos y los intereses privados.

Examinar desde un enfoque comprensivo los diversos registros de la locura invita a considerar el rol que le cupo a la preocupación social respecto de la mente enferma en la sistematización del pensamiento alienista. Evidencia que la medicina y, sobre todo, las instituciones asilares no lograron rivalizar con el protagonismo social en el manejo de las enfermedades mentales y en la discusión respecto de sus características y signos. La delimitación de estrategias para enfrentar sus consecuencias continuó desarrollándose, como ocurriera desde la constitución de la República florentina, sin que fuera necesaria la intervención del conocimiento o acción médicas. Ello no implica que la locura no fuera considerada una enfermedad, o que se considerara que la medicina no poseía el poder de curarla. Por un lado, el hospital de Santa Dorotea fue concebido como un espacio de curación y formación médica donde se exploraron 
una serie de alternativas terapéuticas que resultarían vitales para el desarrollo del pensamiento psiquiátrico posterior, sistematizado por figuras como Vincenzo Chiarugi (Boccadoro y Zandri, 1989, Conti, 2015, Huertas y del Cura, 2004). Por otro lado, tanto los escritos de médicos florentinos como Antonio Cocchi (1685-1758) o Giovanni Targioni Tozzetti (1712-1783) como los registros de Santa Dorotea atestiguan el creciente interés de la comunidad médica Toscana por el problema de la enfermedad mental ${ }^{24}$.

He propuesto aquí un enfoque metodológico que aborda los espacios de la locura interconectando sus distintos objetivos, lenguajes y actores mediante una labor de seguimiento e interconexión de fuentes de distinta naturaleza que, al ser contrastadas entre sí, dan cuenta de aspectos que de otra manera no resultarían accesibles. Aproximarse al estudio de la locura teniendo en consideración las vinculaciones que existian entre las distintas instancias en que se debatió sobre sus características, se evaluaron sus consecuencias y se elaboraron estrategias para hacerle frente entrega una imagen más rica y completa sobre la forma en que fueron concebidas y enfrentadas las perturbaciones mentales en el siglo XVIII. Permite dar cuenta de los recursos con que contaba la sociedad toscana del período, la forma en que eran utilizados, el rol de autoridades, familiares, cercanos o vecinos y las formas en que interactuaban. Pone de manifiesto, también, que el entramado de respuestas se conformaba en base a un diálogo entre intereses públicos y privados que da cuenta de que la locura fue entendida como un problema de responsabilidad social y no solo privada. Por último, al operar con una escala temporal amplia, revela que una misma persona podía pasar por distintas instancias mientras sus parientes probaban diversas opciones para lidiar con las manifestaciones de su enfermedad conforme esta evolucionara, pero que en general se trata de personas que en el largo plazo pudieron permanecer insertas en la vida social, conformar su propia familia y tener un desempeño laboral y social dentro de los márgenes de lo considerado normal.

\section{AGRADECIMIENTOS}

Proyecto CONICYT/FONDECYT Posdoctorado № 3180684. La escritura de este artículo ha sido posible gracias al Fondo Posdoctoral del Programa de Apoyo a la Investigación de la Universidad Adolfo Ibáñez, 2017.

\section{NOTAS}

1. Esto queda de manifiesto, por ejemplo, al observar las tablas de contenidos de revistas especializadas como Social History of Medicine, Medical History, Asclepio, História, Ciências, Saúde-Manghinhos, History of Psiquiatry, entre otras. Por lo demás, la investigación se sigue centrando mayoritariamente en los siglos XIX y XX. Para una mirada panorámica, ver https://historypsychiatry.com/.

2. Este artículo se basa en los resultados de mi tesis doctoral. Mariana Labarca, "Itineraries and Languages of Madness. Family Experience, Legal Practice and Medical Knowledge in Eighteenth-Century Tuscany" (Tesis de Doctorado en Historia, European University Institute, 2015). La investigación fue posible gracias al financiamiento de Becas Chile-Conicyt.

3. Durante el siglo XVIII estos casos eran debatidos principalmente ante dos instancias: el tribunal de justicia criminal (Otto di Guardia e Balia) y la oficina del Auditore Fiscale. Jefe de policía y supervisor de la justicia criminal, este último era una de las tres máximas autoridades de la Consulta (Tribunal de Gracia y de Justicia) y participaba en el Consejo de Regencia. Los registros se encuentran en Archivio di Stato di Firenze (ASF), Consiglio di Reggenza (CR), F. 754-762 y ASF, Otto di Guardia e Balìa (OGB), series Atti, Suppliche y Straordinarie.

4. ASF, CR, F. 754-762 y ASF, OGB, series Atti, Suppliche y Straordinarie.

5. Estos casos eran resueltos directamente ante el Auditore Fiscale. Las causas se pueden revisar en ASF, CR, F. 754-762 y ASF, OGB, Suppliche y Straordinarie.

6. Maria Domenica Marini, interdicta en marzo de 1743, termina su vida en un convento. ASF, Magistrato dei Pupilli et Adulti (MPAP), F. 2301, no. 171, 1743 y F. 139, 7 junio 1744.

7. Es el caso de Francesca Paoli del Feo. ASF, CR, F. 762, n. $2,1765, \mathrm{~s} / \mathrm{f}$.

8. Por ejemplo, ASF, CR, F. 758, no. 2, 1755, s/f., o ASF, MPAP, F. 2304 , no. 2, 1755.

9. ASF, Santa Dorotea (SD), Motupropri, Rescritti, etc, 1750-1785.

10. Fue el caso del Conde Contignoli de Ferrara. ASF, CR, Fiscale, F. 755, N. 25, 1747.

11. ASF, SD, Memorie, Documenti, F. 23, no. 11.

12. Los médicos y los cirujanos pertenecían a gremios distintos en la época moderna. Mientras los primeros tenían una formación universitaria, los segundos seguían un entrenamiento práctico en anatomía en escuelas de cirugía. Durante el siglo XVIII la cirugía se fue profesionalizando a través de instancias formales de práctica en los hospitales que los especializaba en el tratamiento de las enfermeda- 
des "externas" bajo supervisión médica (Cosmacini, 1994 y Pasta, 2006).

13. El médico permanente entre 1756 y 1788 fue Antonio Lulli, y los médicos de consulta Giovanni Targioni Tozzetti y Lodovico Scutellari. Vincenzo Chiarugi asumió la dirección del hospital en 1785 .

14. "Memorie relative alla fondazione dello Sped. Di S. Dorotea et altri documenti attenenti a detto Spedale". ASF, SD, Memorie, Documenti, F. 23, no. 11, capítulo 10, "Del modo di ricevere i Mentecatti".

15. Los registros de la pazzeria de Santa Maria Nuova se encuentran en ASF, Santa María Nuova, n. 13. Todo lo relativo a los ingresos y altas de pacientes de Santa Dorotea están en el archivo de la institución (ASF, SD).

16. “Copia di Motuproprio di S.M.I. per l'erezione del nuovo Conservatorio di S. Dorotea de 16 novembre 1750", ASF, SD, Memorie, Documenti, F. 23, no. 5, inciso 6, s/f. La traducción es mía.

17. ASF, SD, Motupropri, Rescritti, etc, 1756-1757, F. 2, no 2.

18. "Copia di Motuproprio di S.M.I. per l'erezione del nuovo Conservatorio di S. Dorotea de 16 novembre 1750", ASF, SD, Memorie, Documenti, F. 23, no. 5, inciso 6, s/f y ASF, SD, Motupropri, Rescritti, etc, 1756-1757, F. 2, no. 42.

19. Reforma de los estatutos del Magistrato dei Pupilli, 1473, en Francesca Morandini (1955), "Statuti e ordinamenti dell'Ufficio dei Pupilli et adulti nel periodo della Repubblica

\section{BIBLIOGRAFÍA}

Bartlett, Peter (2001), "Legal Madness in the Nineteenth Century", Social History of Medicine 14 (1), pp. 107-131.

Bartlett, Peter; Wright, David (eds.) (1999), Outside the Walls of the Asylum. The History of Care in the Community 17502000, Londres, The Athlone Press.

Biotti, Vittorio (2002), "I saperi sulla follia a Firenze e nel Granducato di Toscana a metà del Seicento". En: Vittorio Biotti (ed.), É matto e tristo, pazzo e fastidioso. I saperi sulla follia: magistrati, medici e inquisitori a Firenze e negli Stati italiani del '600, Florencia, Nicomp L. E., pp. 55-91.

Boccadoro, Sandro; Zandri, Anna (1989), "L'opera riformatrice di Pietro Leopoldo nell'ordinamento giuridico dell'ospedale S. Maria Nuova di Firenze". En: Ciuffoletti, Zeffiro; Rombai, Leonardo (eds.), La Toscana dei Lorena: riforme, territorio, società, Florencia, L. S. Olschki, pp. 279-310

Cartayrade, Laurent (1997), "Property, Prodigality, and Madness: A Study of Interdiction Records in Eighteenth-Century Paris", Tesis conducente al grado de Doctor en Historia no publicada, Universidad de Maryland.

Conti, Norberto Aldo (2015), "Vincenzo Chiarugi: La Pazzia y el reglamento de Bonifacio en los orígenes de la psiquiatría moderna", Asclepio 67, 2. [en línea] doi: http://dx.doi. org/10.3989/asclepio.2015.23

Correa Gómez, María José (2013), “De la Casa de Orates al Juzgado. Pericia alienista y evaluación judicial de la locura en Santiago de Chile hacia 1860", História, Ciências, Saúde fiorentina (1388-1534)", Archivio storico italiano 113, p. 550 .

20. “Bando. Circa l'Esecuzione de'Privilegi, e Statuti del Magistrato delli Signori Ufiziali de'Pupilli, ed Adulti della Città di Firenze, ottenuto nel Supremo Magistrato del di 28 Gennaio 1717", ASF, Magistrato Supremo, Suppliche, F. 1180, fol. 1311.

21. Esto constituye una particularidad de la documentación toscana, diferenciándose de otros expedientes de interdicción (Cartayrade, 1997, Houston, 2000). Las potencialidades del estudio de la judicialización de la locura y especialmente de los expedientes de interdicción han sido estudiados principalmente para el siglo XIX (Suzuki, 2006, Bartlett, 2001, Nootens, 2007, Correa, 2013). Para un análisis comprensivo de esta historiografía ver Correa 2015.

22. Los registros de Navarretti se encuentran en ASF, MPAP, F. 122, f. 2301, F. 2302, F. 2303, F. 2304. Aparece además en ASF, SD, F. 23. Para seguir el derrotero de Barchesi se puede revisar ASF, MPAP, F. 130, F. 1214, F. 2300, F. 2301. Para un análisis más exhaustivo de ambos casos, ver Labarca, 2015.

23. ASF, MS, Suppliche, F. 1209, ff. 402-411.

24. Antonio Cocchi, Consulti Medici del celebre Antonio Cocchi, Vol. I-II (Bergamo: Vincenzo Antoine, 1791); Biblioteca Nazionale Centrale Firenze, Manoscritti, Le carte di Giovanni Targioni Tozzetti, N. 234, "Consultationes medicae".

- Manguinhos, 20 (2), pp. 571-585 [en línea] http://dx.doi. org/10.1590/S0104-59702013000200012, [consultado el 18/12/2017].

Correa Gómez, María José (2015), "La locura desde los archivos. Chile, 1850-1930", Nuevo Mundo Mundos Nuevos, Coloquios, [en línea], https://journals.openedition.org/ nuevomundo/68043.

Cosmacini, Giorgio (1994), Storia della medicina e della sanità in Italia: Dalla peste Europea alla Guerra Mondiale, 13481918, Roma, Laterza.

Foucault, Michel (2006), History of Madness, Londres, Routledge.

Houston, Robert Allan (2000), Madness and Society in EighteenthCentury Scotland, Oxford, Oxford University Press.

Huertas, Rafael; del Cura, Mercedes (2004), "Chiarugi versus Pinel. La carta abierta de Carlo Livi a Brière de Boismont", Frenia, 4 (2), pp. 109-133.

Labarca, Mariana (2015), "Itineraries and Languages of Madness. Family Experience, Legal Practice and Medical Knowledge in Eighteenth-Century Tuscany", Tesis conducente al grado de Doctor en Historia no publicada, Florencia, European University Institute.

Magherini, Graziella; Biotti, Vittorio (1992), L'isola delle Stinche e i percorsi della follia a Firenze nei secoli XIV-XVIII, Florencia, Ponte alle Grazie. 
Magherini, Graziella; Biotti, Vittorio (1998), "Madness in Florence in $14^{\text {th }}-18^{\text {th }}$ Centuries. Judicial Inquiry and Medical Diagnosis, Care and Custody", International Journal of Law and Psychiatry, 21 (4), pp. 355-368.

Mellyn, Elizabeth W. (2014), Mad Tuscans and Their Families. A History of Mental Disorder in Early Modern Italy, Philadelphia, University of Pennsylvania Press.

Morandini, Francesca (1955), "Statuti e ordinamenti dell'Ufficio dei Pupilli et adulti nel periodo della Repubblica fiorentina (1388-1534)", Archivio storico italiano, 113, pp. 520-551.

Nootens, Thierry (2007), Fous, prodigues et ivrognes: Familles et déviance à Montréal au XIX siècle, Montreal, McGillQueen's University Press.

Pasta, Renato (2006), “L'Ospedale e la città": riforme settecentesche a Santa Maria Nuova", Annali di Storia di Firenze, 1, pp. 83-98 [en línea], doi: http://dx.doi.org/10.13128/ Annali_Stor_Firen-9825

Porter, Roy (1990), Mind-Forg'd Manacles: A History of Madness in England from the Restoration to the Regency, Londres, Penguin Books.

Rabin, Dana (2004), Identity, Crime, and Legal Responsibility in Eighteenth-Century England, Basingstoke, Hampshire y Nueva York, Palgrave Macmillan.

Roscioni, Lisa (2003), II governo della follia. Ospedali, medici e pazzi nell'età moderna, Milano, Bruno Mondadori.
Roscioni, Lisa (2011), "Soin et/ou enfermement? Hôpitaux et folie sous l'Ancien Régime", Genèses 82, pp. 31-51.

Roudinesco, Elisabeth, Canguilhem, Georges, Postel, Jacques, Bing, François (et. al.) (1996), Pensar la locura. Ensayos sobre Michel Foucault, Buenos Aires, Paidós.

Rushton, Peter (1988), "Lunatics and Idiots: Mental Disorder, the Community, and the Poor Law in North-East England 1600-1800", Medical History, 32 (1), pp. 34-50, [en línea] doi: https://doi.org/10.1017/S0025727300047591

Stagnaro, Juan Carlos (coord.) (2015), “Dossier: El nacimiento de la psiquiatría: un movimiento europeo", Asclepio, 67 (2), [en línea], doi: https://doi.org/10.3989/asclepio.2015. v67.i2

Suzuki, Akihito (1991-1992), "Lunacy in Seventeenth- and Eighteenth-Century England: Analysis of Quarter Sessions Records", partes 1 y 2, History of Psychiatry 2, pp. 437-456 y 3, pp. 29-44.

Suzuki, Akihito (1998), "The Household and the Care of Lunatics in Eighteenth-Century London". En: Horden, Peregrine; Smith, Richard (eds.), The Locus of Care: Families, Communities, Institutions, and the Provision of Welfare Since Antiquity, Londres, Nueva York, Routledge, pp. 153-175.

Suzuki, Akihito (2006), Madness at Home: The Psychiatrist, the Patient, and the Family in England, 1820-1860, Berkeley, University of California Press. 